\title{
Multiple Model Adaptive Rank Estimation for Integrated Navigation During Mars Entry
}

\author{
Qiang Xiao, Huimin Fu, Zhihua Wang and Yongbo Zhang \\ (Research Center of Small Sample Technology, Beijing University of Aeronautics and \\ Astronautics, Beijing 100191, China) \\ (E-mail: wangzhihua@buaa.edu.cn)
}

\begin{abstract}
Accurate navigation systems are required for future pinpoint Mars landing missions. A radio ranging augmented Inertial Measurement Unit (IMU) integrated navigation system concept is considered for the Mars entry navigation. The uncertain system parameters associated with the Three Degree-Of-Freedom (3-DOF) dynamic model, and the measurement systematic errors are considered. In order to improve entry navigation accuracy, this paper presents the Multiple Model Adaptive Rank Estimation (MMARE) filter of radio beacons/IMU integrated navigation system. 3-DOF simulation results show that the performances of the proposed navigation filter method, $70.39 \mathrm{~m}$ estimated altitude error and $15.74 \mathrm{~m} / \mathrm{s}$ estimated velocity error, fulfill the need of future pinpoint Mars landing missions.
\end{abstract}

\author{
KEYWORDS \\ $\begin{array}{lll}\text { 1. Mars entry autonomous navigation. } & \text { 2. Uncertain system parameters. } & 3 \text {. Measurement }\end{array}$ \\ systematic errors. 4. Multiple model adaptive rank estimation.
}

Submitted: 6 April 2016. Accepted: 1 August 2016. First published online: 28 September 2016.

1. INTRODUCTION. Mars exploration missions play an important role in future deep space exploration activities, and the number of countries and international organisations, which have begun to focus planetary exploration activities on Mars is increasing. Several future international space missions have as an objective the return of Mars surface samples to the Earth (Lévesque and de Lafontaine, 2007). There are many key scientific goals for Mars explorations in order to deepen the understanding of the solar system formation process and the origin of life, such as the search for water and characterisation of aqueous processes on Mars, the study of mineralogy and weathering of the Martian surface, and the search for preserved biosignatures in Martian rocks (Burkhart et al., 2005; Li et al., 2014). However, most of the preselected target sites for the key scientific goals are located at high elevations on the surface of Mars at close proximity to scientifically interesting terrain (Braun and Manning, 2007). If these sites are surrounded by hazards, the lander needs to be precisely delivered from the Mars entry point (defined as a radius of $3,522 \mathrm{~km}$ from the centre of 
Mars, and an altitude of $125 \mathrm{~km}$ over the surface) to the preselected target site with a $100 \mathrm{~m}$ level through the general Mars Entry, Descent, and Landing (EDL) phase of the mission. Looking at past missions, the landing ellipse of the Viking mission was in the order of several hundred kilometres by adopting an Inertial Measurement Unit (IMU) using dead reckoning navigation mode and an unguided ballistic trajectory, which cannot meet the requirements of future manned Mars landing and sample return missions (Marschke et al., 2008; Wang, 2011). Other past missions (Pathfinder; Mars Exploration Rover, MER; Phoenix) used non-lifting trajectories focused only on safe landing, whose landing ellipses were $200 \mathrm{~km} \mathrm{x} 100 \mathrm{~km}, 80 \mathrm{~km} \times 12 \mathrm{~km}$ and $100 \mathrm{~km}$ x $21 \mathrm{~km}$, respectively (Steinfeldt et al., 2010). The Mars Science Laboratory (MSL), whose landing ellipse is $25 \mathrm{~km}$ x $20 \mathrm{~km}$, was launched in 2011 and landed on Mars in 2012 (Vasavada et al., 2012; Dutta and Braun, 2014). So advanced high-precision autonomous navigation and active aerodynamic lift control are essential and need to be well researched (Kozynchenko, 2011; Fu et al., 2015; Li and Peng, 2011; Hormigo et al., 2008).

During the EDL phase, there are three most significant sources of landing position inaccuracy on Mars (Lévesque, 2006; Prince et al., 2011): firstly, position and velocity errors at the atmospheric entry point; secondly, uncertainties in the Martian atmospheric density and the vehicle aerodynamic characteristics during the Mars entry phase and thirdly, drifts caused by strong winds during the parachute descent phase. So the Mars entry phase is the most important and dangerous period during the EDL phase. In order to address the first two significant sources of error, Mars entry navigation technologies play an important role during the whole of a precise landing mission (Li et al., 2014; Braun and Manning, 2007). However, there are three prerequisites affecting Mars entry navigation accuracy: firstly, an accurate Mars entry dynamic model; secondly, sufficient measurement data from the high precision sensors and thirdly, robust state estimation methods. From previous research $(\mathrm{Fu}$ et al., 2015; Lévesque, 2006), three uncertain parameters, which are Martian atmospheric density, ballistic coefficient and lift-to-drag ratio, influence the accuracy of the Mars entry dynamic model. What is more, because most sensors are blocked by the vehicle's heat shield and plasma sheath, only the IMU is available during the Mars entry phase. Fortunately, it has been found that the plasma sheath around the vehicle has little effect on Ultra-High Frequency (UHF) band (300 3,000MHZ) radio communication (Burkhart et al., 2005). In other words, UHF radio communication can be used in the Mars entry phase to enhance the measurement data. As a result of that, a robust state estimation method is needed to effectively reduce the adverse impact of initial state errors and system model parameter uncertainties, and improve Mars entry navigation accuracy.

In the last decade, in order to overcome the adverse effects of parameter uncertainties on state estimation for Mars entry navigation, some research into state estimation methods has been conducted by researchers in different countries and international organisations. Ely et al. (2001) use a Hierarchical Mixture-of-Experts (HME) filter bank as the Mars entry navigation method, in which the filters are parameterised with various atmospheric density and other vehicle parameters. Dubois-Matra and Bishop (2004) applied a multi-model structure comprised of an Extended Kalman Filter (EKF) bank architecture to address the atmospheric density model uncertainty in the Mars entry precision navigation. A Multiple Model Adaptive Estimation (MMAE) method is adopted for Mars entry navigation in order to deal with the 
Martian atmospheric density high uncertainty by Zanetti and Bishop (2007). In another study (Marschke et al., 2008), the sensor bias and scale factors are extended to the vehicle state and estimated through the MMAE method during the Mars entry phase. Recently, Li et al. (2014) adopted a Modified MMAE to address the atmospheric density model uncertainty using integrated navigation. From previous research mentioned above, it is found that there are four common themes. The first one is that all the research is based on the MMAE method, which uses several EKFs running in parallel. As we know, EKF has two major issues (Li and Peng, 2011; Lévesque, 2006): firstly, complex Jacobin matrix calculation is needed and secondly, first-order linearization of the nonlinear system is used. However, for the Mars entry strongly nonlinear navigation system model, using first-order linearization will lead to truncation errors which cause filter divergence, and there is no guarantee that even second order terms can compensate for such errors (Khairnar et al., 2007). Secondly, the works mainly address the atmospheric density model uncertainty. Nevertheless, apart from this, there are ballistic coefficient and lift-to-drag ratio uncertainties affecting the landing accuracy. Thirdly, the navigation scheme for the Mars entry phase mainly adopts IMU measurement data. Finally, the IMU and radio communication measurement model does not consider measurement systematic errors.

In this context, to solve these problems, a type of radio beacon/IMU integrated navigation method based on Multiple Model Adaptive Rank Estimation (MMARE) for the Mars entry phase is researched. In order to avoid the computation of a Jacobin matrix and truncation errors from linearization, the MMARE uses several Rank Filters (RFs) running in parallel instead of EKFs. Rank filter (RF) is a rank sampling method based on the principle of rank statistics (Fu et al., 2014). Considering uncertainties in the Martian atmospheric density and the vehicle aerodynamic characteristics (ballistic coefficient and lift-to-drag ratio) and measurement systematic errors during the Mars entry phase, the improved Three Degree-Of-Freedom (3-DOF) dynamic model and measurement model are obtained. Based on the improved navigation system model, these problems are addressed through the MMARE. Furthermore, through the property of normal distribution, the number of RFs has been greatly reduced. The rest of this paper is structured as follows. In Section 2, the traditional Mars entry dynamic model and the improved Mars entry dynamic model are introduced. Section 3 defines the navigation measurement model considering measurement systematic errors. The navigation method of the MMARE is designed in Section 4. In Section 5, simulations are described and results are discussed. Concluding remarks are in Section 6.

2. MARS ENTRY DYNAMIC MODEL. A simplified realistic 3-DOF dynamic model of a Mars vehicle defined with respect to the Mars-centred Mars-fixed coordinate system is adopted in this Section (Li et al., 2014; Fu et al., 2015). For the sake of simplicity, there are some common assumptions for the Mars entry dynamics used in the literature (Wang, 2011; Fu et al., 2015). Those assumptions are listed as follows: 1) Mars is a spherical shape and non-rotating; 2) the Martian atmosphere is steady and non-rotating, and its density has an exponential behaviour; 3) the Mars vehicle is a point-mass vehicle with a controllable low lift-to-drag ratio. 
The simplified realistic 3-DOF dynamic model of the Mars vehicle is given by

$$
\begin{gathered}
\dot{r}=v \sin \gamma \\
\dot{\theta}=\frac{v \cos \gamma \sin \psi}{r \cos \lambda} \\
\dot{\lambda}=\frac{v}{r} \cos \gamma \cos \psi \\
\dot{v}=-\left(D^{*}+g_{M} \sin \gamma\right) \\
\dot{\gamma}=\left(\frac{v}{r}-\frac{g_{M}}{v}\right) \cos \gamma+\frac{1}{v} L^{*} \cos \phi \\
\dot{\psi}=\frac{v}{r} \sin \psi \cos \gamma \tan \lambda+\frac{L^{*} \sin \phi}{v \cos \gamma}
\end{gathered}
$$

where $r$ is the distance from the Martian centre to the centre of mass of the Mars entry vehicle, $\theta$ is the longitude, $\lambda$ is the latitude, $v$ is the velocity of the Mars entry vehicle, $\gamma$ is the flight path angle (FPA), $\psi$ is the azimuth angle and $\phi$ is the bank angle.

$g_{M}$ is the Martian gravitational acceleration. Typically, the gravitational acceleration model needs to be more accurate and it can be obtained through the addition of spherical harmonics. Considering the $J_{2}$ item, the accuracy of the gravitational acceleration model can reach $99 \cdot 95 \%$. However, ignoring the $J_{2}$ item, the accuracy of the gravitational acceleration calculated by Newton's inverse square force is roughly $99 \%$. Because of the short duration of the Mars entry phase, the effect of the $J_{2}$ item is negligible and the Newton's inverse square Martian gravitational acceleration is used and given by

$$
g_{M}=\frac{\mu}{r^{2}}
$$

where $\mu=4 \cdot 28283 \times 10^{13} \mathrm{~m}^{3} / \mathrm{s}^{2}$ is the Martian gravitational constant.

$D^{*}$ and $L^{*}$ are defined by

$$
D^{*}=\frac{1}{2} \rho^{*} v^{2} B^{*} \quad L^{*}=\frac{1}{2} \rho^{*} v^{2} B^{*} L / D^{*} \quad B^{*}=\frac{C_{D} S}{m_{v}}
$$

where $B^{*}$ is the ballistic coefficient, $L / D^{*}$ is the lift-to-drag ratio, $C_{D}$ is the aerodynamic drag coefficient, $S$ represents the vehicle reference surface area, and $m_{v}$ is the mass of the Mars entry vehicle. The exponential Martian atmospheric density model $\rho *$ is computed as follows

$$
\rho^{*}=\rho_{0}^{*} \exp \left[-\left(\frac{r-r_{s}}{h_{s}}\right)\right]
$$

where $\rho_{0}^{*}=2 \times 10^{-4} \mathrm{~kg} / \mathrm{m}^{3}$ is the reference density, $r_{s}=3437.2 \mathrm{~km}$ is the reference radial radius of Mars (40 km above surface) and $h_{s}=7500 \mathrm{~m}$ is the atmospheric scale height.

As mentioned above, one of the three significant sources of error is the vehicle aerodynamics and the atmospheric density uncertainties. From past research, the uncertainties are listed below (Spencer and Braun, 1996; Braun et al., 1995): 1) the $3-\sigma$ uncertainty of the aerodynamic drag coefficient $C_{D}$ is $\pm 2 \%$ above Mach 10 and $\pm 10 \%$ below Mach 5, and between Mach 5 and Mach 10, a linearly interpolated value for the drag coefficient uncertainty is considered, furthermore, the distribution of 
$C_{D}$ uncertainty is normal; 2) the uncertainty on the atmospheric density is $\pm 60 \%$ above $100 \mathrm{~km}$, and $\pm 30 \%$ below $75 \mathrm{~km}$, and between $75 \mathrm{~km}$ and $100 \mathrm{~km}$, a linearly interpolated value for density uncertainty is considered, moreover, atmospheric density uncertainty is also a normal distribution; 3 ) the uncertainty of the lift-to-drag ratio is $\pm 10 \%$, and its distribution is normal. From the consideration on the safety and rigour, larger $3-\sigma$ uncertainties are chosen, which are separately $\pm 10 \%$ in aerodynamic drag coefficient, $\pm 60 \%$ in atmospheric density and $\pm 10 \%$ in lift-to-drag ratio during the whole Mars entry phase.

Under this condition, the exponential Martian atmospheric density model in Equation (4) is only an approximation of the true density model. As a result of that, in order to obtain a more accurate Martian atmospheric density model, the deviation in the Martian atmospheric density model must be taken into account. The following relationship based on the work of Lévesque (2006) and Braun et al. (1995) can be formulated as follows

$$
\rho_{0}=\rho_{0}^{*}\left(1+\Delta_{\rho}\right)
$$

where $\rho_{0}$ is the improved reference density, $\Delta_{\rho}$ denotes the percentage of deviation in the improved Martian atmospheric density model and it has a normal distribution.

So the improved Martian atmospheric density model $\rho$ can be obtained as follows

$$
\rho=\rho^{*}\left(1+\Delta_{\rho}\right)
$$

Then applying the same process to the density model, the improved ballistic coefficient model and lift-to-drag ratio model can be respectively formulated as follows

$$
\begin{gathered}
B=B^{*}\left(1+\Delta_{B}\right) \\
L / D=L / D^{*}\left(1+\Delta_{L / D}\right)
\end{gathered}
$$

where $B$ is the improved ballistic coefficient, $\Delta_{B}$ denotes the percentage of deviation in the ballistic coefficient model with normal distribution, $L / D$ is the improved lift-todrag ratio, $\Delta_{L / D}$ denotes the percentage of deviation in the lift-to-drag ratio model and its distribution is also normal.

Under these conditions, the improved drag and lift accelerations can be obtained

$$
\begin{gathered}
D=\frac{1}{2} \rho v^{2} B \\
L=\frac{1}{2} \rho v^{2} B L / D
\end{gathered}
$$

Taking Equations (6)-(8) into Equations (9) and (10), the following equations can be obtained

$$
\begin{gathered}
D=\left(1+\Delta_{\rho}\right)\left(1+\Delta_{B}\right) D^{*}=\left(1+\Delta_{\rho}+\Delta_{B}+\Delta_{\rho} \Delta_{B}\right) D^{*} \\
L=\left(1+\Delta_{\rho}\right)\left(1+\Delta_{B}\right)\left(1+\Delta_{L / D}\right) L^{*} \\
=\left(1+\Delta_{\rho}+\Delta_{B}+\Delta_{L / D}+\Delta_{\rho} \Delta_{B}+\Delta_{\rho} \Delta_{L / D}+\Delta_{B} \Delta_{L / D}+\Delta_{\rho} \Delta_{B} \Delta_{L / D}\right) L^{*}
\end{gathered}
$$


Neglecting second-order small terms and higher small term, Equations (11) and (12) can be rewritten as

$$
\begin{gathered}
D=\left(1+\Delta_{\rho}+\Delta_{B}\right) D^{*} \\
L=\left(1+\Delta_{\rho}+\Delta_{B}+\Delta_{L / D}\right) L^{*}
\end{gathered}
$$

Based on the property of normal distribution, Equations (13) and (14) have the following relationship

$$
\begin{aligned}
& D=\left(1+\Delta_{D}\right) D^{*} \\
& L=\left(1+\Delta_{L}\right) L^{*}
\end{aligned}
$$

where $\Delta_{D}=\Delta_{\rho}+\Delta_{B}$ and $\Delta_{L}=\Delta_{D}+\Delta_{L / D}$ are both normal distributions.

Given that, the $3-\sigma$ uncertainties of $\Delta_{D}$ and $\Delta_{L / D}$ are $\pm 70 \%$ and $\pm 10 \%$, respectively. Moreover, the number of uncertain parameters is reduced from three $\left(\Delta_{\rho}, \Delta_{B}\right.$ and $\left.\Delta_{L / D}\right)$ to two $\left(\Delta_{D}\right.$ and $\left.\Delta_{L / D}\right)$. As a result of that, the number of RFs used in the following part can be greatly reduced.

Hence, the improved 3-DOF model can be written as

$$
\begin{gathered}
\dot{r}=v \sin \gamma \\
\dot{\theta}=\frac{v \cos \gamma \sin \psi}{r \cos \lambda} \\
\dot{\lambda}=\frac{v}{r} \cos \gamma \cos \psi \\
\dot{v}=-\left(D+g_{M} \sin \gamma\right) \\
\dot{\gamma}=\left(\frac{v}{r}-\frac{g_{M}}{v}\right) \cos \gamma+\frac{1}{v} L \cos \phi \\
\dot{\psi}=\frac{v}{r} \sin \psi \cos \gamma \tan \lambda+\frac{L \sin \phi}{v \cos \gamma}
\end{gathered}
$$

So the above dynamic model is rewritten with the process noise $\boldsymbol{w}$ as follows

$$
\dot{x}=f(\boldsymbol{x})+\boldsymbol{w}
$$

where

$$
f(\boldsymbol{x})=\left[\begin{array}{c}
v \sin \gamma \\
\frac{v \cos \gamma \sin \psi}{r \cos \lambda} \\
\frac{v}{r} \cos \gamma \cos \psi \\
-\left(D+g_{M} \sin \gamma\right) \\
\left(\frac{v}{r}-\frac{g_{M}}{v}\right) \cos \gamma+\frac{1}{v} L \cos \phi \\
\frac{v}{r} \sin \psi \cos \gamma \tan \lambda+\frac{L \sin \phi}{v \cos \gamma}
\end{array}\right]
$$

$\boldsymbol{x}=\left[\begin{array}{llllll}r & \theta & \lambda & v & \gamma & \psi\end{array}\right]^{T}$ denotes the entry vehicle state variable. 
3. NAVIGATION MEASUREMENT MODEL. In this section, a radio beacon/ IMU integrated navigation scheme is adopted to increase the observation information. The new observation information is the two-way range measurement obtained by the radio communication between the vehicle and an orbiter or surface radio beacon, which is pre-set on the Mars surface or the previous Mars rover of the last mission (Williams et al., 2012; Lightsey et al., 2008). The measurement model is shown as follows.

3.1. Inertial measurement units. Non-gravitational acceleration is obtained from the three mutually orthogonal accelerometers of the IMU directly mounted to the vehicle. The measurement model of the acceleration can be formulated as

$$
\tilde{\boldsymbol{a}}^{B}=\boldsymbol{a}^{B}+\boldsymbol{b}_{a}+\boldsymbol{\eta}_{a}
$$

where $\tilde{\boldsymbol{a}}^{B}$ is the accelerometer measurements along the body coordinate frame, $\boldsymbol{a}^{B}$ is the true acceleration along the body coordinate frame, $\boldsymbol{b}_{a}$ is the accelerometer bias, $\boldsymbol{\eta}_{a}$ is the accelerometer noise approximated by additive, zero-mean, uncorrelated Gaussian random variables.

Because the true acceleration $\boldsymbol{a}^{B}$ along the body frame cannot be obtained, instead, the following relationship is used (Li et al., 2014; Lévesque, 2006)

$$
\boldsymbol{a}^{B}=\boldsymbol{T}_{V}^{B} \boldsymbol{a}^{V}
$$

where $\boldsymbol{T}_{V}^{B}$ is the coordinate transformation matrix from the velocity coordinate frame to the body coordinate frame, and its detailed form, which can be seen in references ( $\mathrm{Li}$ et al., 2014; Wang and Xia, 2015), is not repeated here again. $\boldsymbol{a}^{V}=$ $\left[\begin{array}{lll}-D & -L \sin \phi & L \cos \phi\end{array}\right]$ is the accelerometer measurements along the velocity coordinate frame.

Furthermore, the accelerometer bias of IMU is $\left[3 \times 10^{-3}, \quad 3 \times 10^{-3}, \quad 3 \times 10^{-3}\right] \mathrm{m} / \mathrm{s}^{2}$ (Ali et al., 2005; Peng, 2011) used in this paper.

3.2. Range measurement. Using radio communication for the Mars entry phase has been investigated (Boehmer, 1998; Lou et al, 2014; Way et al, 2013). The range measurement provides the distance between the entry vehicle and an orbiter or a surface beacon. However, the output of the range measurement is the pseudorange with measurement systematic errors. The pseudorange can be reconstructed as follows

$$
\begin{gathered}
\tilde{R}=R+b_{R}+v_{R} \\
R=\sqrt{\left(\boldsymbol{r}-\boldsymbol{r}_{i}\right)^{T}\left(\boldsymbol{r}-\boldsymbol{r}_{i}\right)}
\end{gathered}
$$

where $\tilde{R}$ is the pseudorange, $R$ is the true range between the entry vehicle and an orbiter or a surface beacon, $b_{R}$ represents the radio range bias, and it is assumed to be $200 \mathrm{~m}$ (Yu et al., 2015) in this paper, the range noise $v_{R}$ is approximated by additive, zero-mean, uncorrelated Gaussian random variables. $\boldsymbol{r}$ is the position vector of the entry vehicle and $\boldsymbol{r}_{i}$ is the position vector of the $i t h$ orbiter or surface beacon.

Recalling the navigation measurement equations from Equations (19)-(22), the navigation measurement model is defined as

$$
\boldsymbol{z}=h(\boldsymbol{x})+\boldsymbol{v}
$$


where

$$
\begin{aligned}
& \boldsymbol{z}=\left[\begin{array}{c}
\tilde{\boldsymbol{a}}^{B} \\
\tilde{\boldsymbol{R}}
\end{array}\right] \\
& \tilde{\boldsymbol{a}}^{B}=\left[\begin{array}{lll}
\tilde{a}_{1}^{B}, & \tilde{a}_{2}^{B}, & \tilde{a}_{3}^{B}
\end{array}\right]^{T}, \quad \tilde{\boldsymbol{R}}=\left[\begin{array}{llll}
\tilde{R}_{1}, & \tilde{R}_{2}, & \ldots, & \tilde{R}_{m}
\end{array}\right]^{T} \\
& h(\boldsymbol{x})=\left[\begin{array}{l}
\boldsymbol{a}^{B}+\boldsymbol{b}_{a} \\
\boldsymbol{R}+\boldsymbol{b}_{R}
\end{array}\right]
\end{aligned}
$$

where $\boldsymbol{x}$ denotes the entry vehicle state variable defined in Equation (18). $\tilde{\boldsymbol{a}}^{B}$ and $\tilde{\boldsymbol{R}}$ are the accelerometer and pseudorange measurements, respectively. $m$ is the number of orbiters or surface beacons used for integrated navigation. $\boldsymbol{v}$ is the measurement noise.

4. STATISTICAL ESTIMATORS. In this Section, the MMARE using a bank of RFs is proposed for the nonlinear system model with uncertain system parameters and measurement systematic errors during the Mars entry phase. Compared with the Modified MMAE, the MMARE is applied as the autonomous navigation method in this phase.

In engineering practice, a complex engineering environment will introduce uncertain system parameters to the dynamic model and the outputs from measurement sensors have measurement systematic errors, which will degrade the filter accuracy. Hence, to improve the integrated navigation accuracy during the Mars entry phase, this paper proposes a MMARE navigation filter method.

4.1. Navigation model. Based on Equations (18) and (23) described in Sections 2 and 3, the following basic framework of navigation system model for the MMARE, which involves state estimation of a discrete-time nonlinear dynamic system, can be obtained

$$
\begin{gathered}
\boldsymbol{x}_{k+1}=f\left(\boldsymbol{x}_{k}\right)+\boldsymbol{w}_{k} \\
\boldsymbol{z}_{k}=h\left(\boldsymbol{x}_{k}\right)+\boldsymbol{v}_{k}
\end{gathered}
$$

where $\boldsymbol{x}_{k}$ is the system state vector, $\boldsymbol{z}_{k}$ is the measurement vector, $f(\bullet)$ and $h(\bullet)$ are the state transition function and measurement function, respectively, $\boldsymbol{w}_{k}$ is the system noise vector, $\boldsymbol{v}_{k}$ is the measurement noise vector. They are independent Gaussian noise processes and satisfy

$$
\left\{\begin{array}{l}
\mathrm{E}\left[\boldsymbol{w}_{k}\right]=\boldsymbol{0} \\
\operatorname{Cov}\left[\boldsymbol{w}_{k}, \boldsymbol{w}_{j}\right]=\mathrm{E}\left[\boldsymbol{w}_{k} \boldsymbol{w}_{j}^{T}\right]=\boldsymbol{Q}_{k} \delta_{k j} \\
E\left[\boldsymbol{v}_{k}\right]=\boldsymbol{0} \\
\operatorname{Cov}\left[\boldsymbol{v}_{k}, \boldsymbol{v}_{j}\right]=E\left[\boldsymbol{v}_{k} \boldsymbol{v}_{j}^{\mathrm{T}}\right]=\boldsymbol{R}_{k} \delta_{k j} \\
\operatorname{Cov}\left[\boldsymbol{w}_{k}, \boldsymbol{v}_{j}\right]=E\left[\boldsymbol{w}_{k} \boldsymbol{v}_{j}^{\mathrm{T}}\right]=\boldsymbol{0}
\end{array}\right.
$$

4.2. Multiple model adaptive rank estimation. In this part, the MMARE for discrete-time nonlinear stochastic time-varying system with uncertain system parameters and measurement systematic errors is given. Before that, the RF based on reference (Fu et al., 2014) is introduced as follows.

Step 1: Time update

$$
\boldsymbol{x}_{i, k / k-1}=f\left(\boldsymbol{x}_{i, k-1}\right)
$$




$$
\begin{gathered}
\hat{\boldsymbol{x}}_{k / k-1}=\frac{1}{4 n} \sum_{i=1}^{4 n} \boldsymbol{x}_{i, k / k-1} \\
\boldsymbol{P}_{k / k-1}=\frac{1}{\omega} \sum_{i=1}^{4 n}\left\{\left(\boldsymbol{x}_{i, k / k-1}-\hat{\boldsymbol{x}}_{k / k-1}\right)\left(\boldsymbol{x}_{i, k / k-1}-\hat{\boldsymbol{x}}_{k / k-1}\right)^{\mathrm{T}}\right\}+\boldsymbol{Q}_{k-1}
\end{gathered}
$$

Step 2: Measurement update

$$
\begin{gathered}
\boldsymbol{z}_{i, k / k-1}=h\left(\boldsymbol{\chi}_{i, k / k-1}\right) \\
\hat{\boldsymbol{z}}_{k / k-1}=\frac{1}{4 n} \sum_{i=1}^{4 n} \boldsymbol{z}_{i, k / k-1} \\
\boldsymbol{P}_{z z}=\frac{1}{\omega} \sum_{i=1}^{4 n}\left\{\left(\boldsymbol{z}_{i, k / k-1}-\hat{\boldsymbol{z}}_{k / k-1}\right)\left(\boldsymbol{z}_{i, k / k-1}-\hat{\boldsymbol{z}}_{k / k-1}\right)^{\mathrm{T}}\right\}+\boldsymbol{R}_{k} \\
\boldsymbol{P}_{x z}=\frac{1}{\omega} \sum_{i=1}^{4 n}\left\{\left(\boldsymbol{\chi}_{i, k / k-1}-\hat{\boldsymbol{x}}_{k / k-1}\right)\left(\boldsymbol{z}_{i, k / k-1}-\hat{\boldsymbol{z}}_{k / k-1}\right)^{\mathrm{T}}\right\} \\
K_{k}=\boldsymbol{P}_{x z} \boldsymbol{P}_{z z}^{-1} \\
\hat{\boldsymbol{x}}_{k}=\hat{\boldsymbol{x}}_{k / k-1}+K_{k}\left(\boldsymbol{z}_{k}-\hat{\boldsymbol{z}}_{k / k-1}\right) \\
\boldsymbol{P}_{k}=\boldsymbol{P}_{k / k-1}-K_{k} \boldsymbol{P}_{z z} \mathbf{K}_{k}^{\mathrm{T}}
\end{gathered}
$$

where $n$ is the dimension of system state. The rank sampling points (Fu et al., 2014) are given by

$$
\begin{gathered}
\chi_{i, k-1}= \begin{cases}\hat{\boldsymbol{x}}_{k-1}+u_{p_{1}}\left(\sqrt{\boldsymbol{P}_{k-1}}\right)_{i} & i=1, \cdots, n \\
\hat{\boldsymbol{x}}_{k-1}-u_{p_{1}}\left(\sqrt{\boldsymbol{P}_{k-1}}\right)_{i-n} & i=n+1, \cdots, 2 n \\
\hat{\boldsymbol{x}}_{k-1}+u_{p_{2}}\left(\sqrt{\boldsymbol{P}_{k-1}}\right)_{i-2 n} & i=2 n+1, \cdots, 3 n \\
\hat{\boldsymbol{x}}_{k-1}-u_{p_{2}}\left(\sqrt{\boldsymbol{P}_{k-1}}\right)_{i-3 n} & i=3 n+1, \cdots, 4 n\end{cases} \\
\chi_{i, k / k-1}= \begin{cases}\hat{\boldsymbol{x}}_{k / k-1}+u_{p_{1}}\left(\sqrt{\boldsymbol{P}_{k / k-1}}\right)_{i} & i=1, \cdots, n \\
\hat{\boldsymbol{x}}_{k / k-1}-u_{p_{1}}\left(\sqrt{\boldsymbol{P}_{k / k-1}}\right)_{i-n} & i=n+1, \cdots, 2 n \\
\hat{\boldsymbol{x}}_{k / k-1}+u_{p_{2}}\left(\sqrt{\boldsymbol{P}_{k / k-1}}\right)_{i-2 n} & i=2 n+1, \cdots, 3 n \\
\hat{\boldsymbol{x}}_{k / k-1}-u_{p_{2}}\left(\sqrt{\boldsymbol{P}_{k / k-1}}\right)_{i-3 n} & i=3 n+1, \cdots, 4 n\end{cases}
\end{gathered}
$$

where $u_{p_{1}}$ and $u_{p_{2}}$ are standard normal deviator, and the weight for covariance $\omega$ is equal to $2\left(u_{p_{1}}^{2}+u_{p_{2}}^{2}\right)$. The tuning parameters used for filter are $u_{p_{1}}=0.4823$ and $u_{p_{2}}=$ $1 \cdot 1281$ (Fu et al., 2014).

Given that, as shown in Figure 1, the MMARE is designed with three modules.

The specific three modules are described below:

Module 1: Parallel filter

In this module, a bank of RFs is run in parallel. Here we only give the main parts used in the next two modules, and the other detailed parts can be seen in Equations (30)-(41).

$$
\tilde{\boldsymbol{z}}_{k}^{j}=\boldsymbol{z}_{k}-\hat{\boldsymbol{z}}_{k / k-1}^{j}
$$




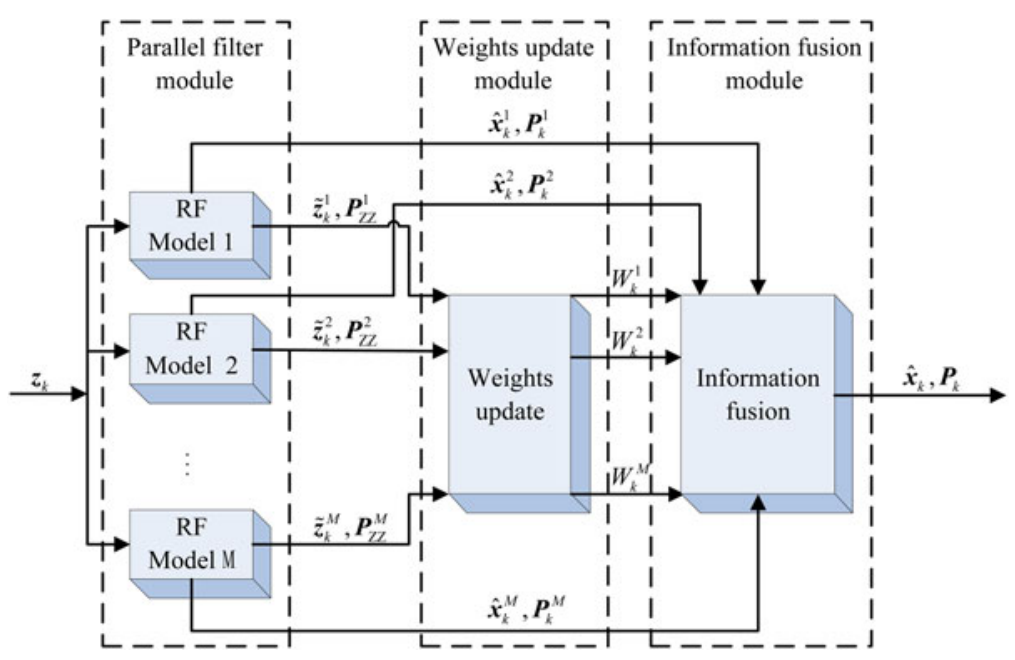

Figure 1. Overview of the multiple model adaptive rank estimation.

$$
\begin{gathered}
\boldsymbol{P}_{z z}^{j}=\frac{1}{\omega} \sum_{i=1}^{4 n}\left\{\left(\boldsymbol{z}_{i, k / k-1}^{j}-\hat{\boldsymbol{z}}_{k / k-1}^{j}\right)\left(\boldsymbol{z}_{i, k / k-1}^{j}-\hat{\boldsymbol{z}}_{k / k-1}^{j}\right)^{\mathrm{T}}\right\}+\boldsymbol{R}_{k} \\
\hat{\boldsymbol{x}}_{k}^{j}=\hat{\boldsymbol{x}}_{k / k-1}^{j}+K_{k}^{j}\left(\boldsymbol{z}_{k}-\hat{\boldsymbol{z}}_{k / k-1}^{j}\right) \\
\boldsymbol{P}_{k}^{j}=\boldsymbol{P}_{k / k-1}^{j}-K_{k}^{j} \boldsymbol{P}_{z z}^{j} K_{k}^{j \mathrm{~T}}
\end{gathered}
$$

where $j=1, \quad \cdots, \quad M$ and $M$ is the number of the RFs. The variables with the superscript $j$ indicate that they are obtained from the $j t h \mathrm{RF}$ in the kth step.

Module 2: Weights update

$$
W_{k}^{j}=\frac{W_{k-1}^{j} \Lambda_{k}^{j}}{\sum_{j=1}^{M} W_{k-1}^{j} \Lambda_{k}^{j}}
$$

where

$$
\Lambda_{k}^{j}=\frac{1}{\sqrt{\left|2 \pi \boldsymbol{P}_{z z}^{j}\right|}} \exp \left[-\frac{1}{2}\left(\tilde{\boldsymbol{z}}_{k}^{j}\right)^{\mathrm{T}}\left(\boldsymbol{P}_{z z}^{j}\right)^{-1} \tilde{\boldsymbol{z}}_{k}^{j}\right]
$$

Module 3: Information fusion

$$
\begin{gathered}
\hat{\boldsymbol{x}}_{k}=\sum_{j=1}^{M} W_{k}^{j} \hat{\boldsymbol{x}}_{k}^{j} \\
\boldsymbol{P}_{k}=\sum_{j=1}^{M} W_{k}^{j}\left[\boldsymbol{P}_{k}^{j}+\left(\hat{\boldsymbol{x}}_{k}-\hat{\boldsymbol{x}}_{k}^{j}\right)\left(\hat{\boldsymbol{x}}_{k}-\hat{\boldsymbol{x}}_{k}^{j}\right)^{\mathrm{T}}\right]
\end{gathered}
$$


The initialisation of the MMARE is given as follows:

$$
\hat{\boldsymbol{x}}_{0}^{j}=\hat{\boldsymbol{x}}_{0}, \quad \boldsymbol{P}_{0}^{j}=\boldsymbol{P}_{0}, \quad W_{0}^{j}=\frac{1}{M}
$$

5. SIMULATION AND RESULTS. To confirm the validity of the MMARE method with uncertain system parameters and measurement systematic errors during the Mars entry phase in this paper, computer simulations and analysis have been carried out by using the MATLAB/Simulink environment. The simulation sample period/step is set to $0.5 \mathrm{~s}$ and the planned entry span is supposed to be $400 \mathrm{~s}$ during the Mars entry phase. To ensure the accuracy and reliability of simulation results, the integral differential equations use a Fourth-order Runge-Kutta algorithm. Firstly, the true state variables with process noise are produced by improved Mars entry dynamic model with uncertain system parameters in an open loop without estimation in order to establish the true trajectory. Secondly, the measurement information is generated from the observation sensors. Finally, the navigation filter using radio beacons/IMU integrated navigation scheme is set up based on the MMARE or Modified MMAE iterative computation, respectively. The navigation errors equal to the state estimation subtracting the true state variables are contrastingly analysed in this section.

One key step of the MMARE is to determine the suitable number of the filters to construct the filter bank. Based on the research in reference (Li et al., 2014), in our simulations, we assume that the MMARE with fifteen independent RFs is enough to perform the integrated navigation. Each filter in the bank is built on a special dynamic model with difference drag acceleration deviations and lift-to-drag ratio deviations shown in Tables 1 and 2. As mentioned in Section 2, because the maximum deviations of the Martian atmospheric density, ballistic coefficient and lift-to-drag ratio are respectively $\pm 60 \%, \pm 10 \%$ and $\pm 10 \%$ compared with real observed data, the deviations all roughly follow a normal distribution around the nominal values. Using the property of normal distribution, through simplification and consolidation, the three deviations are reduced to drag acceleration deviation and lift-to-drag ratio deviation with $\pm 70 \%$ and $\pm 10 \%$ maximum deviations, respectively. Therefore, the drag acceleration deviation $-70 \%,-35 \%, 0,+35 \%$ and $+70 \%$ and the lift-to-drag ratio deviation $-10 \%, 0$ and $+10 \%$ are respectively included into the fifteen different dynamic models in our simulations. Furthermore, given the past research (Lévesque, 2006; Spencer and Braun, 1996; Braun et al., 1995), the true Martian atmospheric density, ballistic coefficient and lift-to-drag ratio are assumed to be respectively $30 \%, 5 \cdot 5 \%$ and $9 \cdot 5 \%$ larger than the nominal values in our simulations.

The initial simulation conditions of the Mars entry vehicle are summarised in Table 3. The physical parameters of the Mars entry vehicle used in this simulation are set as follows: $B^{*}=0.016 \mathrm{~m}^{2} / \mathrm{kg}, L / D^{*}=0.156$ (Lévesque and de Lafontaine, 2007). Furthermore, the number of radio beacons used for the navigation filter has been analysed by Lévesque and de Lafontaine (2007) and Pastor et al. (2000). Based on the conclusions of the past research, three radio beacons are adopted in this paper. The inertial positions of three surface beacons are listed in Table 4 (Lévesque and de Lafontaine, 2007). The radio beacon/IMU integrated navigation filter parameters are shown in Table 5 (Li et al., 2014). 
Table 1. Five dynamic models with different drag acceleration deviation $\Delta_{D}$.

\begin{tabular}{lccccc}
\hline Dynamic model set & Model 1 & Model 2 & Model 3 & Model 4 & Model 5 \\
\hline Deviation $\Delta_{D}$ & $-70 \%$ & $-35 \%$ & 0 & $35 \%$ & $70 \%$ \\
\hline
\end{tabular}

Table 2. Three dynamic models with different lift-to-drag ratio deviation $\Delta_{L / D}$.

\begin{tabular}{lccc}
\hline Dynamic model set & Model 1 & Model 2 & Model 3 \\
\hline Deviation $\Delta_{L / D}$ & $-10 \%$ & 0 & $10 \%$ \\
\hline
\end{tabular}

Table 3. Initial entry conditions (true state) and initial estimator conditions (estimated state).

\begin{tabular}{lcc}
\hline State variables & True & Estimated \\
\hline Initial altitude $h_{0}$ & $125 \mathrm{~km}$ & $126 \mathrm{~km}$ \\
Initial velocity $v_{0}$ & $6900 \mathrm{~m} / \mathrm{s}$ & $6910 \mathrm{~m} / \mathrm{s}$ \\
Initial flight path $\gamma_{0}$ & $-12 \mathrm{deg}$ & $-13 \mathrm{deg}$ \\
Initial azimuth $\psi_{0}$ & $89 \mathrm{deg}$ & $90 \mathrm{deg}$ \\
Initial longitude $\theta_{0}$ & $0 \cdot 00 \mathrm{deg}$ & $0 \cdot 02 \mathrm{deg}$ \\
Initial latitude $\lambda_{0}$ & $1 \cdot 00 \mathrm{deg}$ & $1 \cdot 02 \mathrm{deg}$ \\
\hline
\end{tabular}

Table 4. Position (longitude and latitude) of the reference surface beacons.

\begin{tabular}{lcr}
\hline Beacon type & Initial longitude & Initial latitude \\
\hline Surface beacon 1 & $0 \mathrm{deg}$ & $0 \mathrm{deg}$ \\
Surface beacon 2 & $5 \cdot 7 \mathrm{deg}$ & $5 \cdot 7 \mathrm{deg}$ \\
Surface beacon 3 & $-5 \cdot 7 \mathrm{deg}$ & $5 \cdot 7 \mathrm{deg}$ \\
\hline
\end{tabular}

Using the measurement data from the accelerometers and radio range for the navigation filter, the state estimation errors are computed by the Modified MMAE and MMARE. Each state estimation error from radio beacon/IMU integrated navigation is shown in Figure 2. We can clearly see that the errors of all states obtained from the MMARE are almost smaller than those obtained from the Modified MMAE. As to the states of velocity, FPA and azimuth which are influenced by the uncertain system parameters, it is also found that the three state estimation errors of the MMARE are much smaller. Furthermore, each state estimation Root Mean Squared Error (RMSE) of the Modified MMAE and MMARE is calculated from 500 independent trials and plotted in Figure 3. This shows that the accuracy of the MMARE is the higher of the two filter methods. Compared with the Modified MMAE, the MMARE can much better compensate the negative influences of the uncertain system parameters and measurement systematic errors, and enhance the integrated navigation accuracy. It is further found that the convergence speed of the MMARE is faster than that of the Modified MMAE.

In addition, the mean and covariance of the estimators are calculated from the 500 independent trials and summarised in Table 6. From this Table, it is further found that the RMSE mean and covariance of the MMARE in FPA and azimuth are obviously 
Table 5. IMU/ radio beacons integrated navigation filter parameters.
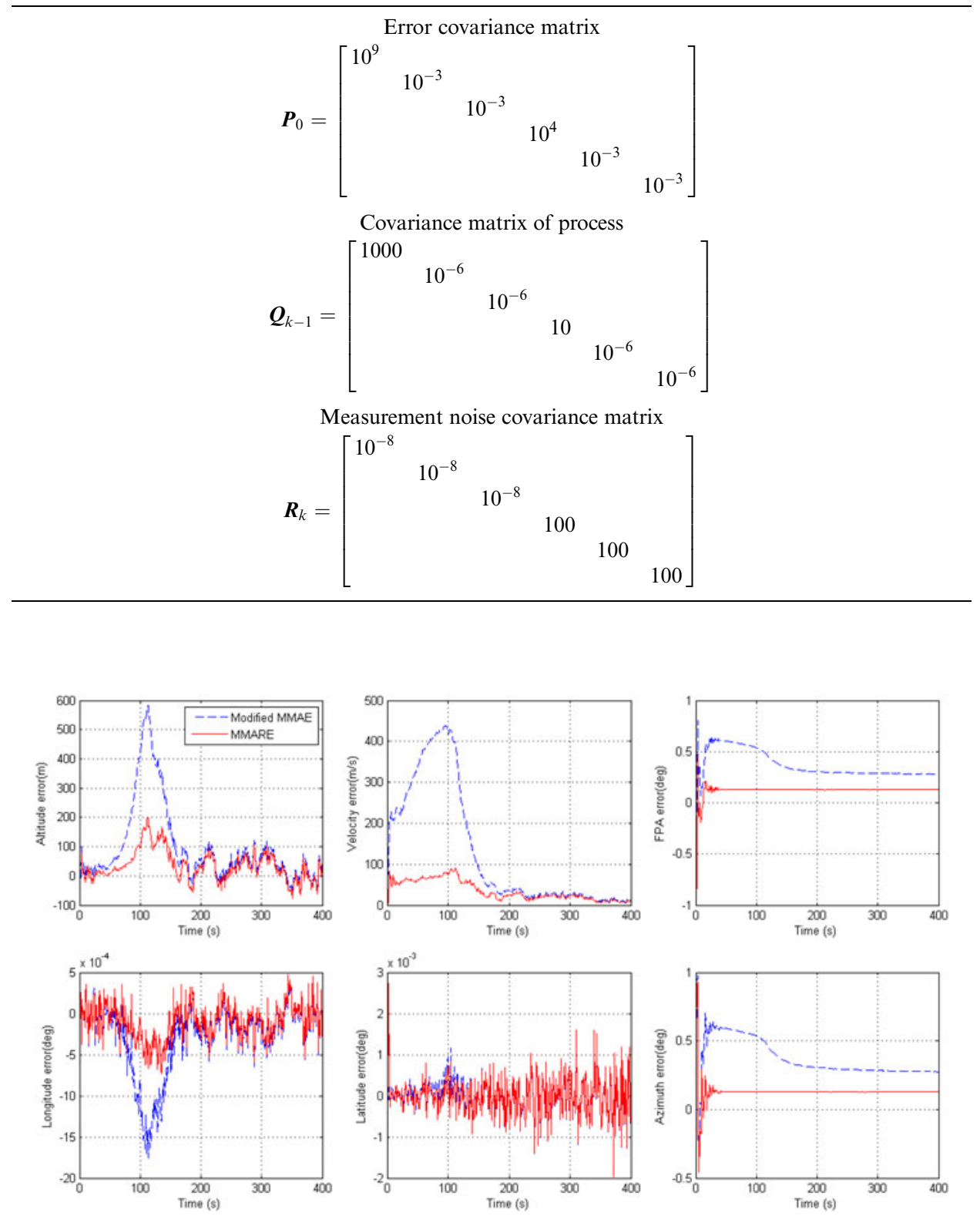

Figure 2. State estimation errors: Modified MMAE-and MMARE- based radio beacons/IMU integrated navigation.

smaller than those of the Modified MMAE, and the FPA and azimuth estimation accuracies of the MMARE are respectively increased by $64 \cdot 49 \%$ and $62 \cdot 41 \%$. With respect to the altitude and velocity estimation accuracy which are important navigation indices, the altitude estimation RMSE of the MMARE reaches $61.15 \mathrm{~m}$, and the estimation accuracy is increased by $47 \cdot 38 \%$ compared with the Modified 

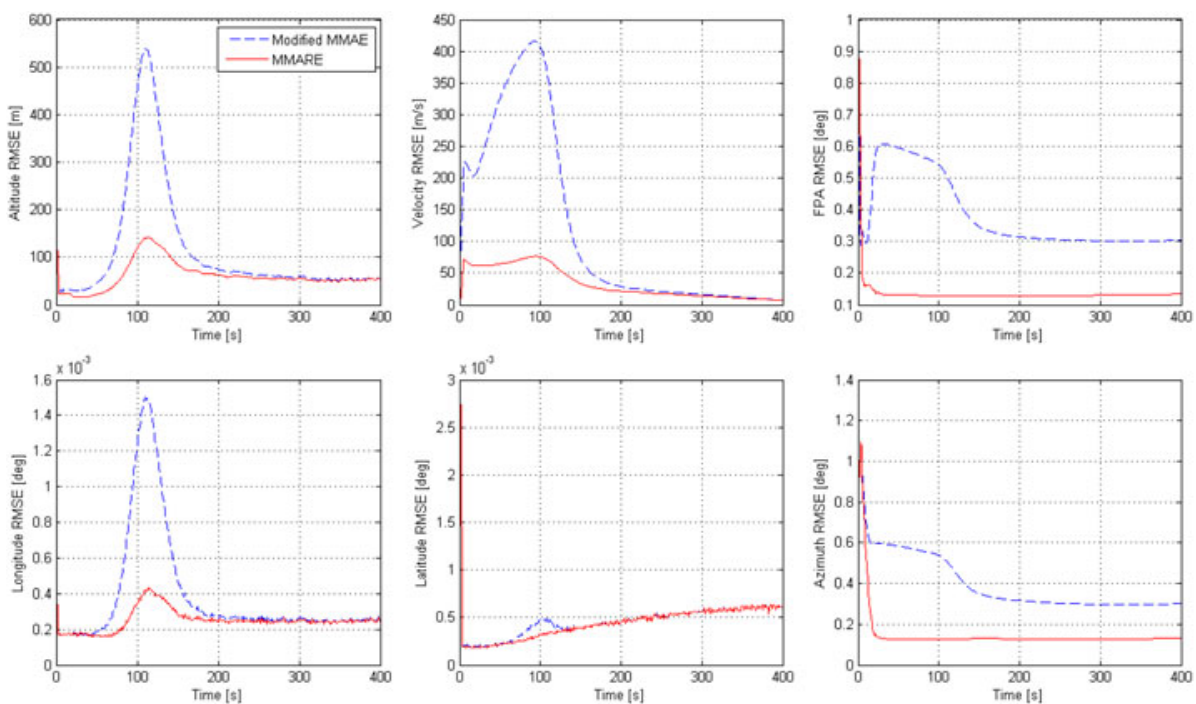

Figure 3. State estimation RMSE: Modified MMAE-and MMARE- based radio beacons/IMU integrated navigation.

Table 6. Performance comparison of the Modified MMAE and MMARE.

\begin{tabular}{|c|c|c|c|c|}
\hline \multirow[b]{2}{*}{ State } & \multicolumn{2}{|c|}{ Modified MMAE } & \multicolumn{2}{|c|}{ MMARE } \\
\hline & RMSE mean & RMSE covariance & RMSE mean & RMSE covariance \\
\hline Altitude(m) & $116 \cdot 21$ & $16038 \cdot 94$ & $61 \cdot 15$ & $864 \cdot 07$ \\
\hline Velocity (m/s) & $119 \cdot 60$ & $20096 \cdot 67$ & 33.64 & $574 \cdot 72$ \\
\hline FPA(deg) & $3.83 \times 10^{-1}$ & $1 \cdot 34 \times 10^{-2}$ & $1.36 \times 10^{-1}$ & $3.08 \times 10^{-3}$ \\
\hline Longitude(deg) & $3.89 \times 10^{-4}$ & $1.05 \times 10^{-7}$ & $2 \cdot 51 \times 10^{-4}$ & $3.69 \times 10^{-9}$ \\
\hline Latitude(deg) & $4.48 \times 10^{-4}$ & $1.74 \times 10^{-8}$ & $4.41 \times 10^{-4}$ & $3 \cdot 57 \times 10^{-8}$ \\
\hline Azimuth(deg) & $3.99 \times 10^{-1}$ & $2 \cdot 07 \times 10^{-2}$ & $1.50 \times 10^{-1}$ & $1.40 \times 10^{-2}$ \\
\hline
\end{tabular}

MMAE. Furthermore, the velocity estimation RMSE reaches $33.63 \mathrm{~m} / \mathrm{s}$, and the estimation accuracy is increased by $71 \cdot 87 \%$.

In order to further confirm the validity of the MMARE method, we have completed another extremely adverse environment simulation. In this environment, the true Martian atmospheric density, ballistic coefficient and lift-to-drag ratio are assumed to be respectively $55 \%, 9 \cdot 8 \%$ and $9 \%$ larger than the nominal values, and the other conditions remain unchanged.

With these conditions, using the radio beacon/IMU integrated navigation scheme, all state estimation errors obtained from Modified MMAE and MMARE are plotted in Figure 4. Furthermore, through 500 independent trials, each state estimation RMSE of the MMARE and Modified MMAE is calculated and plotted in Figure 5. From Figures 4 and 5, we show that the state estimation accuracy of the MMARE is higher than that of the Modified MMAE. It is also found that compared with the Modified MMAE, the MMARE has a faster convergence speed. Tables 6 and 7 summarise the mean and covariance of the two estimators calculated from the 500 

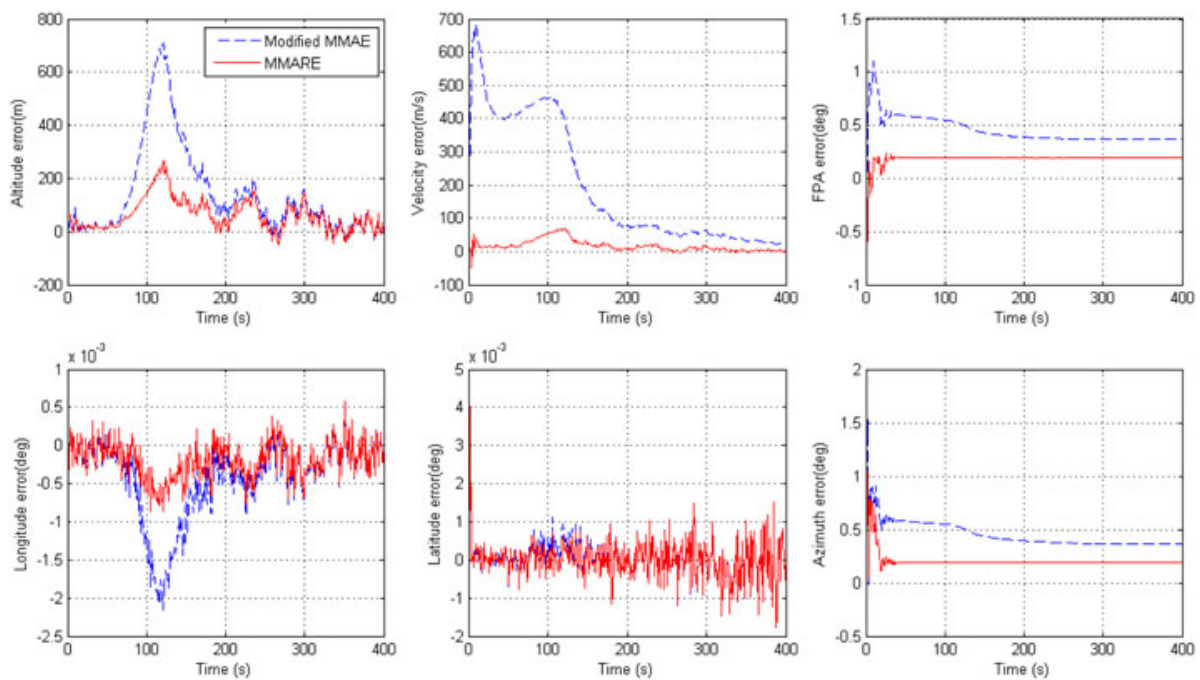

Figure 4. State estimation errors: Modified MMAE-and MMARE- based radio beacons/IMU integrated navigation.
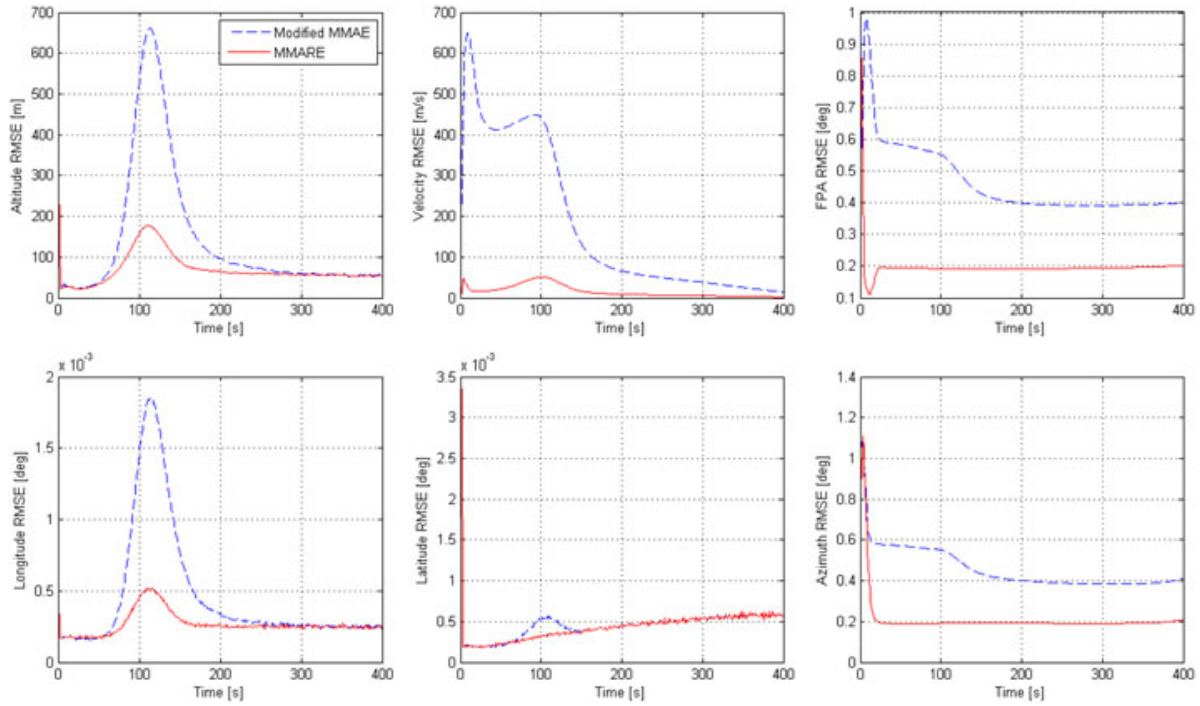

Figure 5. State estimation RMSE: Modified MMAE-and MMARE- based radio beacons/IMU integrated navigation.

independent trials. From Table 7, we further show that the RMSE mean and covariance of the MMARE in FPA and azimuth affected by uncertain system parameters are obviously smaller than those of the Modified MMAE, and the FPA and azimuth estimation errors of the MMARE are respectively decreased by $57 \cdot 85 \%$ and $54 \cdot 25 \%$. With respect to the important navigation index which contains altitude 
Table 7. Performance comparison of the Modified MMAE and MMARE.

\begin{tabular}{lccccc}
\hline & \multicolumn{2}{c}{ Modified MMAE } & & \multicolumn{2}{c}{ MMARE } \\
\cline { 2 - 3 } \cline { 5 - 6 } State & RMSE mean & RMSE covariance & & RMSE mean & RMSE covariance \\
\hline Altitude $(\mathrm{m})$ & $142 \cdot 64$ & $27322 \cdot 37$ & & 70.39 & $1490 \cdot 66$ \\
Velocity $(\mathrm{m} / \mathrm{s})$ & 179.38 & $34364 \cdot 34$ & & $15 \cdot 74$ & $201 \cdot 20$ \\
FPA(deg) & $4.65 \times 10^{-1}$ & $1.29 \times 10^{-2}$ & & $1.96 \times 10^{-1}$ & $2.55 \times 10^{-3}$ \\
Longitude(deg) & $4.64 \times 10^{-4}$ & $1.91 \times 10^{-7}$ & & $2.68 \times 10^{-4}$ & $6.60 \times 10^{-9}$ \\
Latitude(deg) & $4.42 \times 10^{-4}$ & $1.57 \times 10^{-8}$ & & $4.31 \times 10^{-4}$ & $4.11 \times 10^{-8}$ \\
Azimuth(deg) & $4.59 \times 10^{-1}$ & $1 \cdot 15 \times 10^{-2}$ & & $2 \cdot 10 \times 10^{-1}$ & $1 \cdot 11 \times 10^{-2}$ \\
\hline
\end{tabular}

and velocity estimation accuracy, the altitude estimation RMSE of the MMARE reaches $70.39 \mathrm{~m}$, and the estimation accuracy is increased by $50.65 \%$ compared with the Modified MMAE. Furthermore, the velocity estimation RMSE reaches $15 \cdot 74$ $\mathrm{m} / \mathrm{s}$, and the estimation accuracy is increased by $91 \cdot 23 \%$. The other two state estimation accuracies are also slightly improved.

Through the two simulations on different model parameter uncertainties, the MMARE can much better compensate the negative influences of the uncertain system parameters and measurement systematic errors, and enhance the navigation accuracy of the radio beacon/IMU integrated navigation, especially the altitude, velocity, FPA and azimuth estimation accuracies. In other words, the effects of the uncertain system parameters and measurement systematic errors cannot be well reduced by the Modified MMAE for the state estimation so the results obtained by the Modified MMAE are worse than those by the MMARE, even though integrated navigation is used.

6. CONCLUSIONS. In this paper, the MMARE method has been proposed to address the problems of Mars entry navigation with uncertain system parameters and measurement systematic errors. In two simulations with different model parameter uncertainties, Mars entry navigation simulations have shown that the MMARE-based navigation system mitigates the effects due to the uncertain system parameters and measurement systematic errors much better than the Modified MMAE-based navigation system. Even in the extremely adverse Mars entry environment, the estimated altitude and velocity errors reach $70.39 \mathrm{~m}$ and $15.74 \mathrm{~m} / \mathrm{s}$, respectively, for the MMARE-based system. The achieved performances are commensurate with the needs of future pinpoint Mars landing missions.

\section{FINANCIAL SUPPORT}

The work described in this paper was supported by National Basic Research Program of China (grant number 2012CB720000).

\section{REFERENCES}

Ali, K.S., Vanelli, C.A., Biesiadecki, J.J., Biesiadecki, J.J., Maimone, M.W., Cheng, Y., San Martin, A.M. and Alexander, J.A. (2005). Attitude and Position Estimation on the Mars Exploration Rovers. IEEE International Conference on Systems, Man and Cybernetics, Waikoloa, Hawaii. 
Boehmer, R.A. (1998). Navigation Analysis and Design for Mars Entry. Master Thesis, Massachusetts Institute of Technology, Massachusetts, USA.

Braun, R.D. and Manning, R.M. (2007). Mars Exploration Entry, Descent and Landing Challenges. Journal of Spacecraft and Rockets, 44, 310-313.

Braun, R.D., Powell, R.W., Engelund, W.C., Gnoffo, P.A., Weilmuenster, K.J. and Mitcheltree, R.A. (1995). Mars Pathfinder Six-Degree-of-Freedom Entry Analysis. Journal of Spacecraft and Rockets, 32, 993-1000.

Burkhart, P.D., Ely, T., Duncan, C., Lightsey, E.G., Campbell, T. and Mogensen, A. (2005). Expected EDL Navigation Performance with Spacecraft to Spacecraft Radiometric Data. AIAA Guidance, Navigation, and Control Conference, San Francisco, CA.

Dubois-Matra, O. and Bishop, R.H. (2004). Multi-Model Navigation with Gating Networks for Mars Entry Precision Landing. AIAA Atmospheric Flight Mechanics Conference and Exhibit, Providence, Rhode Island.

Dutta, S. and Braun, R.D. (2014). Statistical Entry, Descent, and Landing Performance Reconstruction of the Mars Science Laboratory. Journal of Spacecraft and Rockets, 51, 1048-1061.

Ely, T.A., Bishop, R.H. and Dubois-Matra, O. (2001). Robust Entry Navigation Using Hierarchical Filter Architectures Regulated with Gating Networks. 16th International Symposium on Spaceflight Dynamics Symposium, Pasadena, CA.

Fu, H.M., Xiao, Q. and Wu, Y.Z. (2014). Rank Filter Method. Journal of Mechanical Strength, 36, 521-526.

Fu, H.M., Yang, Y.S., Xiao, Q., Wu, Y.Z. and Zhang, Y.B. (2015). Autonomous Navigation Method Based on Unbiased Minimum-Variance Estimation during Mars Entry. Advances in Space Research, 55, 1512 1528.

Hormigo, T., Silva, J.A. and Câmara, F. (2008). Nonlinear Dynamic Inversion-Based Guidance and Control for a Pinpoint Mars Entry. AIAA Guidance, Navigation and Control Conference and Exhibit, Honolulu, Hawaii.

Khairnar, D.G., Nandakumar, S., Merchant, S.N. and Desai, U.B. (2007). Nonlinear Target Identification and Tracking Using UKF. IEEE International Conference on Granular Computing, Fremont, CA.

Kozynchenko, A.I. (2011). Analysis of Predictive Entry Guidance for a Mars Lander under High Model Uncertainties. Acta Astronautica, 68, 121-132.

Lévesque, J.F. (2006). Advanced Navigation and Guidance for High-Precision Planetary Landing on Mars. $\mathrm{PhD}$ thesis, Université de Sherbrooke, Sherbrooke, Canada.

Lévesque, J.F. and de Lafontaine, J. (2007). Innovative Navigation Schemes for State and Parameter Estimation during Mars Entry. Journal of Guidance, Control, and Dynamics, 30, 169-184.

Li, S., Jiang, X.Q. and Liu, Y.F. (2014). Innovative Mars Entry Integrated Navigation Using Modified Multiple Model Adaptive Estimation. Aerospace Science and Technology, 39, 403-413.

Li, S. and Peng, Y.M. (2011). Radio Beacons/IMU Integrated Navigation for Mars Entry. Advances in Space Research, 47, 1265-1279.

Lightsey, E.G., Mogensen, A.E., Burkhart, P.D., Ely, T.A. and Duncan, C. (2008). Real-Time Navigation for Mars Missions Using the Mars Network. Journal of Spacecraft and Rockets, 45, 519-533.

Lou, T.S., Fu, H.M., Wang, Z.H. and Zhang, Y.B. (2014). Schmidt-Kalman Filter for Navigation Biases Mitigation during Mars Entry. Journal of Aerospace Engineering, 28, 1-7.

Marschke, J.M., Crassidis, J.L. and Lam, Q.M. (2008). Multiple Model Adaptive Estimation for Inertial Navigation during Mars Entry. AIAA/AAS Astrodynamics Specialist Conference and Exhibit, Honolulu, Hawaii.

Peng, Y.M. (2011). Innovative Navigation Guidance and Control Technology for Mars Entry Descent and Landing. Master Thesis, Nanjing University of Aeronautics and Astronautics, Nanjing, China.

Prince, J.L., Desai, P.N. and Queen, E.M. (2011). Mars Phoenix Entry, Descent, and Landing Simulation Design and Modeling Analysis. Journal of Spacecraft and Rockets, 48, 756-764.

Pastor, P.R., Gay, R.S., Striepe, S.A. and Bishop, R.H. (2000). Mars Entry Navigation from EKF Processing of Beacon Data. AIAAIAAS Astrodynamics Specialist Conference, Denver, CO.

Spencer, D.A. and Braun, R.D. (1996). Mars Pathfinder Atmospheric Entry: Trajectory Design and Dispersion Analysis. Journal of Spacecraft and Rockets, 33, 670-676.

Steinfeldt, B.A., Grant, M.J., Matz, D.A., Braun, R.D. and Barton, G.H. (2010). Guidance, Navigation, and Control System Performance Trades for Mars Pinpoint Landing. Journal of Spacecraft and Rockets, 47, 188-198.

Vasavada, A.R., Chen, A., Barnes, J.R., Burkhart, P.D., Cantor, B.A., Dwyer-Cianciolo, A.M., Fergason, R. L., Hinson, D.P., Justh, H.L., Kass, D.M., Lewis, S.R., Mischna, M.A., Murphy, J.R., Rafkin, S.C.R., 
Tyler, D. and Withers, P.G. (2012). Assessment of Environments for Mars Science Laboratory Entry, Descent, and Surface Operations. Space Science Reviews, 170, 793-835.

Wang, X.C. and Xia, Y.Q. (2015). Navigation Strategy with the Spacecraft Communications Blackout for Mars Entry. Advances in Space Research, 55, 1264-1277.

Wang, Y.B. (2011). Research on Autonomous Navigation Method for Mars Atmospheric Entering. Master Thesis, Harbin Institute of Technology, Harbin, China.

Way, D.W., Davis, J.L. and Shidner, J.D. (2013). Assessment of the Mars Science Laboratory Entry, Descent, and Landing Simulation. AAS/AIAA 23rd Space Flight Mechanics Meeting, Kauai, HI.

Williams, J.L., Menon, P.R. and Demcak, S.W. (2012). Mars Reconnaissance Orbiter Navigation Strategy for Mars Science Laboratory Entry, Descent and Landing Telecommunication Relay Support. AIAA/ AAS Astrodynamics Specialist Conference, Minneapolis, Minnesota.

Yu, Z.S., Cui, P.Y. and Zhu, S.Y. (2015). Observability-Based Beacon Configuration Optimization for Mars Entry Navigation. Journal of Guidance, Control, and Dynamics, 38, 643-650.

Zanetti, R. and Bishop, R.H. (2007). Adaptive Entry Navigation Using Inertial Measurements. AAS/AIAA Space Flight Mechanics Meeting, Sedona, AZ. 